\title{
EDITORIAL •ÉDITORIAL
}

\section{Leçons tirées de la pandémie de COVID-19 (à ce jour)}

Les opinions exprimées dans cet éditorial sont celles de l'auteur et ne représentent pas nécessairement celles de l'Association médicale canadienne ou ses filiales.

a pandémie de maladie à coronavirus 2019 (COVID-19) a bouleversé la vie de tous et chacun. Les pratiques médicales ont été chamboulées et la vie de la population générale a subi d'extrêmes transformations. Il est facile de voir le côté négatif de toute chose, or, beaucoup d'éléments positifs ont aussi été mis en lumière.

Il est clair à présent que c'est la science qui guide les décisions. Et si on ignore la science, la société y perd. Les valeurs fondamentales qui sous-tendent la science de qualité - la transparence et la collaboration l'emporteront. Sitôt connue, la structure génétique du coronavirus du syndrome respiratoire aigu sévère 2 , le virus responsable de la COVID-19, a immédiatement été accessible et les pays se sont empressés de subventionner la recherche et d'assurer un marché pour les vaccins afin que les laboratoires puissent se pencher sur des solutions. Même à présent, les petits laboratoires qui se font distancer par la concurrence, acceptent de créer des partenariats avec d'autres fabricants plus avancés sur la voie de la commercialisation afin d'assurer un approvisionnement approprié en vaccin. Des centaines d'essais ont rapidement déployé des protocoles randomisés prospectifs pour générer des preuves scientifiques claires et tuer dans l'œuf les prétentions avancées par des charlatans. La base de données de l'Organisation mondiale de la santé montre que 3547 essais ont été enregistrés auprès de ClinicalTrials.gov en 1 an pour une seule maladie. La science se trouve ainsi en terrain inconnu. Les agences subventionnaires gouvernementales et privées ont soutenu de tout leur poids économique les efforts de recherche sur la COVID-19. Presque toutes les revues scientifiques ont traité en priorité la recherche sur la COVID-19 et permis aux articles à ce sujet de prendre le pas sur d'autres à des fins de publication. Dans bien des cas, les frais de publication et d'examen des données ont été suspendus. Au Fournal canadien de chirurgie, nous avons priorisé les articles sur la COVID-19, en publiant des résultats préliminaires et de nouveaux protocoles pour faire notre part de toutes les façons possibles.

En ces temps difficiles, nous avons aussi pris conscience de nos faiblesses. Certaines dataient évidemment d'avant la pandémie. Ainsi, dans plusieurs secteurs manufacturiers, nous avons laissé l'expertise quitter le
Canada. Pour la fabrication des vaccins, nous ne disposions plus d'aucune infrastructure. Le Canada prévoit à présent fabriquer des vaccins, avec le Programme fédéral d'aide aux infrastructures. Comme les 2 établissements prévus ne seront pas prêts avant la fin de 2021 et le début de $2023^{1}$. Il sera probablement trop tard pour la pandémie actuelle. Curieusement, le gouvernement fédéral a fait un bien piètre boulot pour ce qui est de se procurer des vaccins pour le Canada. Tout en s'assurant en théorie que le Canada reçoive des vaccins à raison de 5 fois les taux requis per capita, nous tirons de l'arrière par rapport à d'autres pays pour ce qui est des taux de vaccination ${ }^{2}$. Pourquoi est-il si difficile pour nous de planifier sur une période de 8 mois ce qu'il faudrait faire si nous avions 10000,100000 ou 1 million de doses ou plus? On dirait que les gouvernements fédéral et provinciaux/territoriaux ont été dépassés et n'ont pu réfléchir aux options et préparer à l'avance un plan de déploiement. Même nos centres hospitaliers locaux ont connu de grandes variations quant aux calendriers vaccinaux pour le personnel soignant à risque. La santé publique a besoin d'une refonte - et non d'injections d'argent inconsidérées, comme dans un puits sans fond. Cette crise nous apprend que les personnes en charge de la gestion quotidienne de notre réponse à la pandémie sont dépassées. Le manque de collaboration entre les gouvernements fédéral et provinciaux/territoriaux est à pleurer, avec des conférences de presse quotidiennes au cours desquelles ils se renvoient la balle. Nul ne peut prédire quand nous sortirons de cette crise. Nous devrions être en train de planifier et de construire puisque, selon toute vraisemblance, cette pandémie n'est pas la dernière. C'est l'occasion rêvée pour remettre les pendules à l'heure. Nous allons faire l'expérience d'un cycle économique difficile, peu importe la suite des événements. Nous devons nous assurer que les ressources que nous utilisons pour nous reconstruire sont durables, que les solutions sont à long terme et que nous ne faisons pas que mettre des diachylons ou éteindre des incendies. La communication devra assurément être plus harmonieuse entre les paliers fédéral et provinciaux/territoriaux.

Affiliations : Coéditeur en chef, fournal canadien de chirurgie; Département de chirurgie, Université McGill, Montréal, Qué. 
Intérêts concurrents : E.J. Harvey est médecin chef chez Greybox Solutions; il est cofondateur et directeur de l'innovation médicale de NXTSens Inc, cofondateur et médecin chef de MY01 Inc. et cofondateur et directeur de Strathera Inc. Il bénéficie du soutien institutionnel de J \& J, DePuy Synthes, Stryker et Zimmer.

Propriété intellectuelle du contenu : Il s'agit d'un article en libre accès distribué conformément aux modalités de la licence Creative Commons Attribution (CC BY-NC-ND 4.0), qui permet l'utilisation, la diffusion et la reproduction dans tout médium à la condition que la publication originale soit adéquatement citée, que l'utilisation se fasse à des fins non commerciales (c.-à-d., recherche ou éducation) et qu'aucune modification ni adaptation n'y soit apportée. Voir : https://creativecommons.org/licenses/by-nc-nd/4.0/ deed.fr.

DOI: $10.1503 /$ cjs.003921

\section{Références}

1. Walsh M, Semeniuk I, Kirkup K. Canada to start domestic production months after COVID-19 vaccine deadline. Globe and Mail [Toronto] 2016; le 29 février. Disponible: https://www. theglobeandmail.com/politics/article-canada-to-start-domestic -production-months-after-covid-19-vaccine/ (consulté le 3 février 2021).

2. Tasker JP. Canada falls to 20th in the world for vaccine doses administered. CBC News 2021 Jan. 28. Disponible: https:// www.cbc.ca/news/politics/canada-20-world-vaccine-doses -administered-1.5891465 (consulté le 3 février 2021). 